\title{
AN ALTERNATE METHOD OF DERIVING PRIORITIES AND RELATED INFERENCES FOR GROUP DECISION MAKING IN ANALYTIC HIERARCHY PROCESS
}

\author{
Indrani Basak \\ Department of Mathematics and Statistics \\ Penn State Altoona \\ Altoona, PA, USA \\ E-mail: i8b@psu.edu
}

\begin{abstract}
Some work has been done in the past on statistically deriving priorities in Analytic Hierarchy Process (AHP). In AHP, the aggregated worths of the alternatives, when compared with respect to several criteria, are estimated in a hierarchical comparisons model introduced by Saaty. In this setup, statistical models are used for Saaty's method of scaling in paired comparisons experiments in any level of the hierarchy. At the end, the final priority weights of the alternatives and related inferences are developed with appropriate statistical methods. Existing statistical methods in the literature assume independence of the entries of the paired comparison matrix. However these entries are highly dependent among themselves. In this article, we propose a statistical method which allows for the dependence among the entries of the pairwise comparisons matrix. The proposed method is then illustrated with a numerical example. We also consider the interval estimation based on large-sample theory and examine the actual coverage probabilities of these confidence intervals in case of small samples using a Monte Carlo simulation study.
\end{abstract}

Keywords: aggregated priority weight, dependence of judgments, equality of two priority vectors, maximum likelihood estimate, rank order.

\section{Introduction}

Most of the paired comparison experiments involve preference of one alternative to another on the basis of several criteria. In the most of the situations, one needs an aggregated estimate of the priority weights of a set of alternatives with respect to overall quality when compared under several criteria. This multi-criteria decision making has drawn attention from many research workers. The Analytic Hierarchy Process (AHP; (Saaty, 1980)) is one such methodology which has received substantial application in various fields. In the AHP, the priority weights of the alternatives with respect to each criterion can be determined and then aggregated to give a single overall priority weight of an alternative through a hierarchical structure. Setting up a hierarchy of interrelated elements (alternatives and criteria) is an important aspect of AHP. Once the hierarchy is constructed, decision maker(s) provides(provide) his/her (their) relative preferences for the different alternatives by way of a series of pairwise comparisons in each level of the hierarchy. A matrix of pairwise comparisons is formed from these judgments for a decision maker.

The eigenvalue method is a deterministic (non-stochastic) method to derive priority weights. In this method, the errors in judgments are assumed not to exist. However, the true priority weights of the alternatives are difficult to be assessed by the decision makers in various real life decision making problems. Hence, it is natural to assume that the pairwise comparisons provided by the decision makers are subject to random error. In that light, development of probabilistic (stochastic) formulation of the AHP is relevant and has attracted considerable interest [for example, (Arbel, 1989), (Basak, 1991), (Basak, 1998), (Bryson and Joseph, 2000), (Crawford and Williams, 1985), (de Jong, 1984), (Genest and Rivest, 1994), (Hahn, 2003), (Haines, 1998), (Jensen,, 1984), (Lipovetsky and Tishler, 1999), (Ramanathan. 1997), (Saaty and Vargas, 1987), (Stam and Duarte Silva, 1997), (Sugihara, Ishii, and Tanaka, 2004)]. Stochastic method 
is of much relevance when the priority weights are to be derived for a group of decision makers. In that situation, stochastic formulation is established through the variability across the multiple decision makers in which case the random errors for these individuals are assumed to have a known probability distribution. Random errors for different paired comparison entries, however, are generally assumed to be independent. But in reality these random errors are clearly dependent in the view of the definition of consistency $\left(a_{i k}=a_{i j} \times a_{j k}\right)$. This defintion of consistency is not necessarily satisfied for all $i, j, k=1,2, \cdots, t$ where $t$ is the total number of alternatives and thus it gives rise to an inconsistent pairwise comparison matrix. In any (consistent or inconsistent) pairwise comparison matrix, one of the entries out of $a_{i k}, a_{i j}$ and $a_{j k}$ is connected with the other two. Thus, the stochastic methods which are based on the assumption of independence among the entries of the pairwise comparison matrix are not reliable, especially when one makes some statistical inferences. It is the objective of this article to allow for the dependence among the pairwise comparison entries in a stochastic method of deriving priority weights.

In section 2, a suitable statistical model is proposed which is not only simple and intuitive but also allows for the dependence of the pairwise comparison entries. Relevant statistical inferences are proposed in section 3 for the statistical model developed in section 2. Aggregation of the priority weights through the hierarchical structure to generate a single overall priority weight of an alternative is discussed in section 4 along with relevant statistical inference. Finally, the stochastic method developed in the article is illustrated by some numerical examples and simulation studies in section 6 .

\section{The Statistical Model}

The complex system of the preference problem is decomposed into smaller subsystems in different levels of a hierarchy. In a simplest form of a hierarchy consisting of three levels, the top of the hierarchy is the overall objective of making the best comparisons. The second level consists of the criteria for preference. The final level of the hierarchy contains the alternatives.

In group decision making, a number of individuals participate in providing judgments. They compare the priority weights of the criteria with respect to the over-all suitability as well as the alternatives with respect to each single criterion. Suppose $t$ alternatives $T_{i} ; i=1,2, \cdots, t$ are compared with respect to $m$ criteria $C_{\alpha} ; \alpha=1,2, \cdots, m$. Let us denote the priority weights of the $t$ alternatives when compared according to the $\alpha$-th criterion by the vector $\boldsymbol{\pi}^{\alpha}$ where $\boldsymbol{\pi}^{\alpha \prime}=\left(\pi_{1}^{\alpha}, \pi_{2}^{\alpha}, \cdots, \pi_{t}^{\alpha}\right)$ and the priority weights of the $m$ criteria by the vector $\boldsymbol{\pi}$ where $\boldsymbol{\pi}^{\prime}=\left(\pi_{1}, \pi_{2}, \cdots, \pi_{m}\right) \cdot \sum_{i=1}^{t} \pi_{i}^{\alpha}=1$ for all $\alpha$ and $\sum_{\alpha=1}^{m} \pi_{\alpha}=1$.

Priority weights of the second level are combined with those of the third level to produce the over-all priority weight vector $\pi^{*}$ of the $t$ alternatives when compared under $m$ criteria is obtained by the equation

$$
\pi^{*}=D \pi
$$

where

$$
\left.\begin{array}{rl}
\boldsymbol{\pi}^{* \prime} & =\left(\pi_{1}^{*}, \pi_{2}^{*}, \cdots, \pi_{t}^{*}\right) \\
\boldsymbol{D} & =\left(\boldsymbol{\pi}^{1}, \boldsymbol{\pi}^{2}, \cdots, \boldsymbol{\pi}^{m}\right) \\
\boldsymbol{\pi}^{\prime} & =\left(\pi_{1}, \pi_{2}, \cdots, \pi_{m}\right) .
\end{array}\right\}
$$

In (2) and henceforth $\mathbf{w}^{\prime}$ denote the transpose of the vector $\mathbf{w}$. Consider a typical situation in a certain level of the hierarchy. $s$ ( $s$ can be t or $\mathrm{m}$ ) elements (elements can be alternatives or criteria) are compared pairwise by a number of individuals. These individuals are chosen so that they have a common ground for their beliefs. In case, individual judgments are so apart that they cannot be reconciled, separate homogeneous groups of individuals can be formed. Let us assume that $N$ individuals form a homogeneous group and $a_{u v k}$ denote the pairwise comparison matrix entry denoting the intensity of preference (in a ratio scale) of alternative $T_{i}$ to alternative $T_{j}$ (or of criterion $C_{\alpha}$ to criterion $C_{\beta}$ ) by the $k$-th individual, 
$k=1,2, \cdots, N$. Since the preferences $a_{u v k}$ are collected in ratio scale, it can be thought to be an estimate of the ratio $p_{u} / p_{v}$ where $\mathbf{p}^{\prime}=\left(p_{1}, p_{2}, \cdots, p_{s}\right)$ is the vector of non-normalised $\left(\sum_{u=1}^{s} p_{u} \neq 1\right)$ priority weights of the alternatives. Although the usual practice is to collect only the pairwise comparison entries above the diagonal (given the reciprocal matrix where $a_{v u}=1 / a_{u v}$ ), we will consider collecting both the entries $a_{u v}$ and $a_{v u}$ for more information and for allowing us to form the statistical model we are proposing in this article. Also in that case the estimation problem is invariant to the indexing of the items because of the fixed entries in both $a_{u v}$ and $a_{v u}$. For example, if one observes $a_{12}$ to be 3 , then if some time gap is allowed before observing $a_{21}$, the later may be observed at some value other than $1 / 3$ even for the same individual. With these $a_{i j k} \mathrm{~s}$, for the $k$-th individual, let $y_{i k}$ denote

$$
y_{u k}=\frac{1}{1+\sum_{v=1, v \neq u}^{s} \frac{1}{a_{u v k}}}
$$

for $u=1,2, \cdots, s, k=1,2, \cdots, N$. It is then clearly shown that $y_{u k}$ can be thought to be an estimate of the ratio $\pi_{u}=p_{u} / \sum_{u=1}^{s} p_{u} ; u=1,2, \cdots, s$.

Thus, one can consider the following stochasic model

$$
y_{u k}=\pi_{u}+e_{u k}
$$

in which $e_{u k} \mathrm{~s}$ are random variables with the expected value (mean) zero for $u=1,2, \cdots, s, k=1,2, \cdots, N$. Most of the body of the existing literature assumes $e_{u k}$ s to be independently distributed. In this article, we relax that assumption and allow for the dependence of the $e_{u k} \mathrm{~s}$. As discussed before, $a_{u v}$ s realistically are not independent and as a consequence $e_{u k}$ s realistically should not be distributed independently. We, therefore, assume that the covariance (dispersion) matrix of $e_{u k} \mathrm{~s}$ to be given by a non-diagonal matrix $\boldsymbol{\Sigma}$. In order to simplify the related statistical inference method we further assume that $e_{u k} \mathrm{~s}$ have Normal distribution. Hence, in a common statistical notation, $e_{u k} \mathrm{~s}$ are distributed as multi-variate Normal $N(0, \boldsymbol{\Sigma})$. From (4), $y_{u k} \mathrm{~s}$ are then distributed as multi-variate Normal $N(\boldsymbol{\pi}, \Sigma)$ where $\boldsymbol{\pi}^{\prime}=\left(\pi_{1}, \pi_{2}, \cdots, \pi_{s}\right)$.

In the next section, we present some appropriate statistical inferences regarding a single group (normalised) priority vector $\boldsymbol{\pi}$. We also present a statistical method for testing the equality of the normalised priority vectors for two groups of individuals in there.

\section{Inference on the Priority Weights}

Let the vector $\mathbf{Y}_{\mathbf{k}}{ }^{\prime}$ denote the vector of the random variables $\left(y_{1 k}, y_{2 k}, \cdots, y_{s, k}\right)$ for the $k$-th individual in a given group; $k=1,2, \cdots, N$ where $y_{u k} ; u=1,2, \cdots, s$ are computed as (3). Then $\mathbf{Y}_{\mathbf{1}}{ }^{\prime}, \mathbf{Y}_{\mathbf{2}}{ }^{\prime}, \cdots, \mathbf{Y}_{\mathbf{N}}{ }^{\prime}$ are independently and identically distributed random vectors from multi-variate Normal $N(\boldsymbol{\pi}, \boldsymbol{\Sigma})$. The maximum likelihood estimates of $\boldsymbol{\pi}$ and $\boldsymbol{\Sigma}$ are given, respectively, by

$$
\mathbf{p}^{\prime}=\left(p_{1}, \cdots, p_{s}\right) ; \quad p_{u}=\frac{\bar{y}_{u N}}{\sum_{u=1}^{N} \bar{y}_{u N}} ; u=1, \cdots, s
$$

where

$$
\overline{\mathbf{Y}}_{N}=\frac{1}{N} \sum_{k=1}^{N} \mathbf{Y}_{\mathbf{k}}=\left(\bar{y}_{1 N}, \cdots, \bar{y}_{s N}\right)
$$

and 


$$
\mathbf{S}_{N}=\frac{1}{N-1} \sum_{k=1}^{N}\left(\mathbf{Y}_{\mathbf{k}}-\overline{\mathbf{Y}}_{N}\right)\left(\mathbf{Y}_{\mathbf{k}}-\overline{\mathbf{Y}}_{N}\right)^{\prime}
$$

\subsection{Testing a Hypothesis That The Normalised Priority Vector $\pi$ Is A Given Vector}

For a small number $N$ of individuals in a group, let the hypothesis that normalised priority vector $\boldsymbol{\pi}$ is a given vector $\boldsymbol{\pi}_{0}$ be denoted by $H_{01}$; i.e, $H_{01}: \boldsymbol{\pi}=\boldsymbol{\pi}_{0}$. The test statistic for testing $H_{01}$ is $T_{1 N}^{2}=$ $N\left(\overline{\mathbf{Y}}_{N}-\boldsymbol{\pi}_{0}\right)^{\prime} \mathbf{S}_{N}^{-1}\left(\overline{\mathbf{Y}}_{N}-\boldsymbol{\pi}_{0}\right)$. The well-known likelihood ratio test rejects $H_{01}$ when $T_{1 N}^{2}$ exceeds the critical value

$$
T_{10}^{2}=\frac{(N-1)(s)}{N-s} F_{s, N-s}(\alpha)
$$

in which $F_{s, N-s}(\alpha)$ is the $100 \alpha \%$ point of the $F$-distribution with the numerator and the denominator degrees of freedom given by $s$ and $N-s$ respectively.

When the number $N$ of individuals in a group is sufficiently large $(N \geq 30)$, then $H_{0}$ is rejected when $T_{1 N}^{2}$ exceeds the critical value $\chi_{s}^{2}$ where $\chi_{s}^{2}$ is the $100 \alpha \%$ point of the $\chi^{2}$-distribution with degrees of freedom $s$.

\subsection{A Confidence Region for the Priority Vector $\pi$}

The probability is $1-\alpha$ of drawing a sample of $N$ with mean $\overline{\mathbf{Y}}_{N}$ and covariance matrix $S_{N}$ such that

$$
N\left(\overline{\mathbf{Y}}_{N}-\boldsymbol{\pi}\right)^{\prime} \mathbf{S}_{N}^{-1}\left(\overline{\mathbf{Y}}_{N}-\boldsymbol{\pi}\right) \leq T_{10}^{2}
$$

in which $T_{10}^{2}$ is given by (7). When (8) is computed for a particular sample, we have confidence probability $1-\alpha$ that (8) is a true statement concerning the vector $\boldsymbol{\pi}$. The inequality in (8) is the interior and boundary of an ellipsoid in the $s$-dimensional space of $\boldsymbol{\pi}$ with center at $\overline{\mathbf{Y}}_{N}$ and with size and shape depending on $\mathbf{S}_{N}^{-1}$ and $\alpha$ and we state that $\boldsymbol{\pi}$ lies within this ellipsoid with confidence probability $1-\alpha$.

\subsection{A Test For The Rank Order In Priority Vector $\pi$}

Using specific simultaneous confidence intervals for linear combinations of the priority vector, one can perform a test for the rank order in the normalised priority vector $\boldsymbol{\pi}$. One can assert with the confidence $1-\alpha$ that the unknown priority vector $\boldsymbol{\pi}$ satisfies simultaneously for all $\gamma$ the inequalities

$$
\left|\boldsymbol{\gamma}^{\prime} \overline{\mathbf{Y}}_{N}-\boldsymbol{\gamma}^{\prime} \boldsymbol{\pi}\right| \leq \sqrt{\boldsymbol{\gamma}^{\prime} \mathbf{S} \gamma} \sqrt{\frac{T_{10}^{2}}{N}}
$$

The confidence region in (8) can be explored by appropriately setting $\gamma$ in (9). For example, by setting $\gamma$ to be $(1,0,0, \cdots, 0)^{\prime}$ and $(0,0,1,0, \cdots, 0)^{\prime}$, one can get the confidence intervals for $\pi_{1}$ and $\pi_{3}$ respectively. For testing the rank order in the priority vector, however, one needs to get the simultaneous confidence intervals for $\pi_{1}-\pi_{2}, \pi_{2}-\pi_{3}, \cdots, \pi_{s-1}-\pi_{s}$. Confidence intervals for $\pi_{1}-\pi_{2}, \pi_{2}-\pi_{3}, \cdots, \pi_{s-1}-\pi_{s}$ are accomplished by choosing the following $\gamma$ vectors of length $(s-1)$ 


$$
\left.\begin{array}{c}
\gamma_{1}=(1,-1,0, \cdots, 0)^{\prime}, \\
\gamma_{2}=(0,1,-1, \cdots, 0)^{\prime}, \\
\vdots \\
\gamma_{s-1}=(0,0, \cdots, 0,1,-1)^{\prime} .
\end{array}\right\}
$$

In that case, using (9) and (10), the simultaneous confidence intervals of $\pi_{1}-\pi_{2}, \pi_{2}-\pi_{3}, \cdots, \pi_{s-1}-\pi_{s}$ turn out to be

$$
\left.\begin{array}{c}
\left(\bar{Y}_{1}-\bar{Y}_{2}-M_{1}, \bar{Y}_{1}-\bar{Y}_{2}+M_{1}\right), \\
\left(\bar{Y}_{2}-\bar{Y}_{3}-M_{2}, \bar{Y}_{2}-\bar{Y}_{3}+M_{2}\right), \\
\vdots \\
\left.-\bar{Y}_{s-1}-M_{s-1}, \bar{Y}_{s-2}-\bar{Y}_{s-1}+M_{s-1}\right)
\end{array}\right\}
$$

where $\overline{\mathbf{Y}}_{N}^{\prime}=\left(\bar{Y}_{1}, \bar{Y}_{2}, \cdots, \bar{Y}_{s-1}\right)$ and $M_{u}=\sqrt{\boldsymbol{\gamma}_{u}^{\prime} \mathbf{S} \gamma_{u}} \sqrt{\frac{T_{10}^{2}}{N}} ; u=1,2, \cdots, s-1$ with $\gamma_{u} ; u=1,2, \cdots, s-1$ as given in (10). If any of the individual confidence intervals includes 0 , then that particular pair of the priority vectors should be considered tied. For example, if the confidence interval for $\pi_{2}-\pi_{3}$, given by the second one in (11), includes 0 , then the priorities $\pi_{2}$ and $\pi_{3}$ would be considered to be tied. On the other hand, if that particular confidence interval consists of all positive (negative) numbers then we conclude that $\pi_{2}$ have significantly higher (lower) rank than $\pi_{3}$. At the end, simultaneously considering all of these individual confidence intervals, one establishes a statistical rank order in the prioirity vector.

\subsection{A Test For The Equality Of The Priority Vectors Of Two Groups}

In many situations, there are two groups of individuals who are interested in the same decision making involving same sets of alternatives and criteria. In that case, a natural question arises about the similarities or dissimilarities of the two priority vectors of these two groups of individuals. Let us denote the (normalized) priority vectors for these two groups by $\boldsymbol{\pi}^{(1)}$ and $\boldsymbol{\pi}^{(2)}$ and let the hypothesis that these priority vectors are equal be denoted by $H_{02}$; i.e, $H_{02}: \boldsymbol{\pi}^{(1)}=\boldsymbol{\pi}^{(2)}$.

The covariance matrices of the populations of these two groups are assumed to be equal but unknown. Let $\mathbf{Y}_{1}^{(1)}, \mathbf{Y}_{2}^{(1)}, \cdots, \mathbf{Y}_{N_{1}}^{(1)}$ denote the vectors of random variables (as defined in the beginning of section 3) from the first group of individuals. Let us assume that these are independently and identically distributed from multi-variate Normal $N\left(\boldsymbol{\pi}_{1}^{(1)}, \boldsymbol{\Sigma}\right)$. Let $\mathbf{Y}_{1}^{(2)}, \mathbf{Y}_{2}^{(2)}, \cdots, \mathbf{Y}_{N_{2}}^{(2)}$ denote those for the second group of individuals and let us assume that these are independently and identically distributed from multi-variate Normal $N\left(\boldsymbol{\pi}^{(2)}, \boldsymbol{\Sigma}\right)$. Then $\overline{\mathbf{Y}}_{N_{1}}^{(1)}$ and $\overline{\mathbf{Y}}_{N_{2}}^{(2)}$ will denote the mean for these two groups and they are distributed as multivariate Normal $N\left(\boldsymbol{\pi}^{(1)}, \boldsymbol{\Sigma} / N_{1}\right)$ and $N\left(\boldsymbol{\pi}^{(2)}, \boldsymbol{\Sigma} / N_{2}\right)$ respectively. Consequently, the test statistic for testing $H_{02}$ is

$$
T_{2 N}^{2}=\frac{N_{1} N_{2}}{N_{1}+N_{2}}\left(\overline{\mathbf{Y}}_{N_{1}}^{(1)}-\overline{\mathbf{Y}}_{N_{2}}^{(2)}\right)^{\prime} \mathbf{S}^{-1}\left(\overline{\mathbf{Y}}_{N_{1}}^{(1)}-\overline{\mathbf{Y}}_{N_{2}}^{(2)}\right)
$$


in which the sample covariance matrix is

$$
\mathbf{S}=\frac{1}{N_{1}+N_{2}-2}\left[\sum_{u=1}^{N_{1}}\left(\overline{\mathbf{Y}}_{u}^{(1)}-\overline{\mathbf{Y}}_{N_{1}}^{(1)}\right)\left(\overline{\mathbf{Y}}_{u}^{(1)}-\overline{\mathbf{Y}}_{N_{1}}^{(1)}\right)^{\prime}+\sum_{u=1}^{N_{2}}\left(\overline{\mathbf{Y}}_{u}^{(2)}-\overline{\mathbf{Y}}_{N_{2}}^{(2)}\right)\left(\overline{\mathbf{Y}}_{u}^{(2)}-\overline{\mathbf{Y}}_{N_{2}}^{(2)}\right)^{\prime}\right]
$$

$H_{02}$ is rejected when $T_{2 N}^{2}$ exceeds the critical value

$$
T_{20}^{2}=\frac{\left(N_{1}+N_{2}-2\right)(s)}{N_{1}+N_{2}-s-1} F_{s, N_{1}+N_{2}-s-1}(\alpha)
$$

in which $F_{s, N_{1}+N_{2}-s-1}(\alpha)$ is the $100 \alpha \%$ point of the $F$-distribution with the numerator and the denominator degrees of freedom given by $s$ and $N_{1}+N_{2}-s-1$ respectively.

Simultaneous confidence intervals of $\boldsymbol{\pi}^{(1)}-\boldsymbol{\pi}^{(2)}=\mathbf{m}$ (say) could be used to compare the priority vectors $\boldsymbol{\pi}^{(1)}$ and $\boldsymbol{\pi}^{(2)}$ of two groups of individuals componentwise and are given by

$$
\left|\gamma^{\prime}\left(\overline{\mathbf{Y}}_{N_{1}}^{(1)}-\overline{\mathbf{Y}}_{N_{2}}^{(2)}\right)-\gamma^{\prime} \mathbf{m}\right| \leq \sqrt{\gamma^{\prime} \mathbf{S} \boldsymbol{\gamma}} \sqrt{\frac{N_{1}+N_{2}}{N_{1} N_{2}} T_{2 N}^{2}}
$$

for all $\boldsymbol{\gamma}$. Selecting length $s$ vectors $\gamma_{1}^{\prime}=(1,0,, \cdots, 0), \gamma_{2}^{\prime}=(0,1,0, \cdots, 0), \cdots, \gamma_{s}^{\prime}=(0,0, \cdots, 0,1)$, one gets simultaneous confidence intervals for the differences of component priorities $\pi_{u}^{(1)}-\pi_{u}^{(2)} ; u=1,2, \cdots, s$. Then simultaneous confidence intervals of $\pi_{1}^{(1)}-\pi_{1}^{(2)}, \pi_{2}^{(1)}-\pi_{2}^{(2)}, \cdots, \pi_{s}^{(1)}-\pi_{s}^{(2)}$ turn out to be

$$
\left.\begin{array}{c}
\left(\bar{Y}_{1}^{(1)}-\bar{Y}_{1}^{(2)}-P_{1}, \bar{Y}_{1}^{(1)}-\bar{Y}_{1}^{(2)}+P_{1}\right), \\
\left(\bar{Y}_{2}^{(1)}-\bar{Y}_{2}^{(2)}-P_{2}, \bar{Y}_{2}^{(1)}-\bar{Y}_{2}^{(2)}+P_{2}\right), \\
\vdots \\
\left(\bar{Y}_{s}^{(1)}-\bar{Y}_{s}^{(2)}-P_{s}, \bar{Y}_{s}^{(1)}-\bar{Y}_{s}^{(2)}+P_{s}\right),
\end{array}\right\}
$$

where $P_{u}=\sqrt{\gamma_{u}^{\prime} \mathbf{S} \gamma_{u}} \sqrt{\frac{N_{1}+N_{2}}{N_{1} N_{2}} T_{20}^{2}} ; u=1,2, \cdots, s$. If any of the individual confidence intervals includes 0 , then that particular component of the priority vectors should be considered tied. For example, if the confidence interval for $\pi_{2}^{(1)}-\pi_{2}^{(2)}$, given by the second one in (14), includes 0 , then the priorities $\pi_{2}^{(1)}$ and $\pi_{2}^{(2)}$ would be considered to be tied. On the other hand, if that particular confidence interval consists of all positive (negative) numbers then we conclude that $\pi_{2}^{(1)}$ have significantly higher (lower) than $\pi_{2}^{(2)}$.

\section{Aggregation of the Priority Weights}

Maximum likelihood procedure described in section 3 generates the vectors $\pi^{1}, \boldsymbol{\pi}^{2}, \ldots \boldsymbol{\pi}^{2}$ as well as the vector $\boldsymbol{\pi}$. These vectors are combined in the composite priority vector $\boldsymbol{\pi}^{*}$ of the alternatives for the overall preference through the equations given by (1) and (2).

The following lemma gives the covariance matrix of the vector $\boldsymbol{\pi}^{*}$. 
Lemma : If the error terms associated with the criteria and alternatives as well as those corresponding to the different criteria are uncorrelated then the covariance matrix $\boldsymbol{\Sigma}^{*}$ of the over-all priority weight vector $\pi^{*}$ is given by

$$
\boldsymbol{\Sigma}^{*}=\left(\begin{array}{cc}
\boldsymbol{\Sigma}_{1} & \mathbf{b} \\
\mathbf{b}^{\prime} & \sigma_{00}
\end{array}\right) .
$$

where $\sigma_{i j}$ is the $i j$-th element of the matrix $\boldsymbol{\Sigma}_{1}$ and is given by

$$
\sigma_{i j}=\operatorname{cov}\left(\pi_{i}^{*}, \pi_{j}^{*}\right)=\sum_{\alpha=1}^{m} \sigma_{i j}^{\alpha}\left[\sigma_{\alpha \alpha}+\left(\pi_{\alpha}\right)^{2}\right]+\sum_{\alpha=1}^{m} \sum_{\beta=1}^{m} \pi_{i}^{\alpha} \sigma_{\alpha \beta} \pi_{j}^{\beta}
$$

for $i, j=1,2, \cdots, t-1$. In (16), $\sigma_{i j}^{\alpha}$ is the $i j$-th element of the covariance matrix $\Sigma^{\alpha}$ of the priority vector of $t$ alternatives with respect to $\alpha$-th criterion and $\sigma_{\alpha \beta}$ is the $\alpha \beta$-th element of the covariance matrix $\Sigma_{\text {criteria }}$ of the priority vector of $m$ criteria. In (15), the vector $\mathbf{b}$ is given by $\mathbf{b}^{\prime}=\left(b_{1}, b_{2}, \cdots, b_{t-1}\right)$ where

$$
b_{i}=-\sum_{j=1}^{t-1} \sigma_{i j}
$$

and $\sigma_{00}$ is given by

$$
\sigma_{00}=\sum_{i=1}^{t-1} \sum_{j=1}^{t-1} \sigma_{i j} .
$$

Proof : A result given in Feldstein (1971) states that if $\boldsymbol{\alpha}, \boldsymbol{\beta}$ and $\boldsymbol{\gamma}$ are random vectors with respective mean vectors $\overline{\boldsymbol{\alpha}}, \overline{\boldsymbol{\beta}}$ and $\overline{\boldsymbol{\gamma}}$, then provided both $\boldsymbol{\alpha}$ and $\boldsymbol{\beta}$ are uncorrelated with $\boldsymbol{\gamma}$,

$$
\operatorname{cov}\left(\boldsymbol{\alpha}^{\prime} \boldsymbol{\gamma}, \boldsymbol{\beta}^{\prime} \boldsymbol{\gamma}\right)=\operatorname{tr}[\operatorname{cov}(\boldsymbol{\gamma}) \operatorname{cov}(\boldsymbol{\beta}, \boldsymbol{\alpha})]+\overline{\boldsymbol{\alpha}}^{\prime} \operatorname{cov}(\boldsymbol{\gamma}) \overline{\boldsymbol{\beta}}+\overline{\boldsymbol{\gamma}}^{\prime} \operatorname{cov}(\boldsymbol{\beta}, \boldsymbol{\alpha}) \overline{\boldsymbol{\gamma}}
$$

where $\operatorname{tr}[\mathrm{A}]$ denotes the trace of matrix $A$. Let now $\boldsymbol{\alpha}$ and $\boldsymbol{\beta}$ in (19) be replaced by $\mathbf{d}_{i}$ and $\mathbf{d}_{j}$, the estimated $i$-th and $j$-th row of the matrix $\boldsymbol{D}$ of (2) and $\boldsymbol{\gamma}$ in (19) be replaced by vector $\mathbf{p}$, the estimate of the priority weight vector (of $m$ criteria) $\boldsymbol{\pi}^{\prime}$ given by (2). Then, one gets

$$
\begin{aligned}
\sigma_{i j} & =\operatorname{cov}\left(\boldsymbol{\pi}_{i}^{*}, \boldsymbol{\pi}_{j}^{*}\right) \\
& =\operatorname{cov}\left(\mathbf{d}_{i}^{\prime} \mathbf{p}, \mathbf{d}_{j}^{\prime} \mathbf{p}\right) \\
& =\operatorname{tr}\left[\operatorname{cov}(\mathbf{p}) \operatorname{cov}\left(\mathbf{d}_{j}, \mathbf{d}_{i}\right)\right]+\overline{\mathbf{d}}_{i}^{\prime} \operatorname{cov}(\mathbf{p}) \overline{\mathbf{d}}_{j}+\overline{\mathbf{p}}^{\prime} \operatorname{cov}\left(\mathbf{d}_{j}, \mathbf{d}_{i}\right) \overline{\mathbf{p}}
\end{aligned}
$$

If the error terms associated with different criteria are uncorrelated, $\operatorname{cov}\left(\boldsymbol{d}_{j}, \boldsymbol{d}_{i}\right)$ is a diagonal matrix with entries $\sigma_{i j}^{1}, \sigma_{i j}^{2}, \cdots, \sigma_{i j}^{m}$. Then,

$$
\operatorname{tr}\left[\operatorname{cov}(\mathbf{p}) \operatorname{cov}\left(\boldsymbol{d}_{j}, \boldsymbol{d}_{i}\right)\right]=\sum_{\alpha=1}^{m} \sigma_{i j}^{\alpha} \sigma_{\alpha \alpha}
$$

Next it can be seen easily that

$$
\overline{\mathbf{d}}_{i}^{\prime} \operatorname{cov}(\mathbf{p}) \overline{\mathbf{d}}_{j}=\boldsymbol{\pi}_{i}^{\prime} \operatorname{cov}(\boldsymbol{p}) \boldsymbol{\pi}_{j}
$$




$$
=\sum_{\alpha=1}^{m} \sum_{\beta=1}^{m} \pi_{i}^{\alpha} \sigma_{\alpha \beta} \pi_{j}^{\beta}
$$

and

$$
\begin{aligned}
\overline{\mathbf{p}}^{\prime} \operatorname{cov}\left(\mathbf{d}_{j}, \mathbf{d}_{i}\right) \overline{\mathbf{p}} & =\boldsymbol{\pi}^{\prime} \operatorname{cov}\left(\mathbf{d}_{j}, \mathbf{d}_{i}\right) \boldsymbol{\pi} \\
& =\sum_{\alpha=1}^{m}\left(\pi_{\alpha}\right)^{2} \sigma_{i j}^{\alpha} .
\end{aligned}
$$

Finally, substituting the results of (21), (22) and (23) in the right hand side of (20), one gets (16). Also, (17) and (18) follow after noting that

$$
\sum_{i=1}^{t} \pi_{i}^{*}=1
$$

Remark: In order to get the estimates of $\sigma_{i j}$ in (16), one needs to use the estimate of the covariance

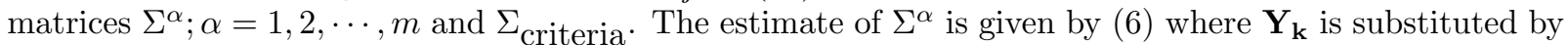
$\left(y_{1 k}^{\alpha}, y_{2 k}^{\alpha}, \cdots, y_{t-1, k}^{\alpha}\right)$. Here, $y_{i k}^{\alpha} ; i=1,2, \cdots, t-1$ is obtained by (3) with $s$ replaced by $t$ and $a_{u v k}$ replaced by $a_{i j k}^{\alpha}$, the $(i, j)$-th pairwise comparison entry under criterion $\alpha$ by the $k$-th individual.

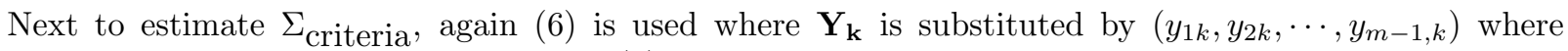
$y_{\alpha k}, \alpha=1,2, \cdots, m-1$ is obtained by (3) with with $s$ replaced by $m$ and $a_{u v k}$ replaced by $a_{\alpha \beta k}$, the pairwise comparison entry of criterion $\alpha$ and $\beta$ by the $k$-th individual. Let then (3) is denoted by $S_{N}^{(1)}$. The estimate of $\Sigma_{\text {criteria }}$ is given by

$$
\left(\begin{array}{cc}
\boldsymbol{S}_{N}^{(1)} & \mathbf{d} \\
\mathbf{d}^{\prime} & s_{0}
\end{array}\right)
$$

In $(24)$, the vector $\mathbf{d}$ is given by $\mathbf{d}^{\prime}=\left(d_{1}, d_{2}, \cdots, d_{m-1}\right)$ where

$$
d_{i}=-\sum_{j=1}^{m-1} s_{i j}^{(1)}
$$

and $s_{0}$ is given by

$$
s_{0}=\sum_{i=1}^{m-1} \sum_{j=1}^{m-1} s_{i j}^{(1)}
$$

where $s_{i j}^{(1)}$ is the $i j$-th element of the matrix $\boldsymbol{S}_{N}^{(1)}$.

\section{Numerical Illustration}

In this section, we present an artificial data on the school selection example given by (Saaty, 1980) to illustrate the method developed in the paper. Seven independent observations for each of the thirty pairs of six criteria [Learning (L), Friends (F), School Life (S), Vocational Training (V), College Preparatiopn (C) and Music Classes (M)] were collected from seven judges of similar background in an hypothetical experiment. The data are recorded in Table 1. For example, the fourth observation on the pair (L,F) is 1.5 which means that $\mathrm{L}$ is preferred 1.5 times $\mathrm{F}$. Also, three schools $(\mathrm{A}, \mathrm{B}, \mathrm{C})$ were compared with respect to each of these six criteria. The data are recorded in Tables 2 through 7. 
Table 1. Comparisons Data of six criteria with respect to overall satisfaction with school; $N=7$.

\begin{tabular}{|c|ccccccc|}
\hline & \multicolumn{7}{|c|}{ Observations } \\
\cline { 2 - 8 } Pairs & 1 & 2 & 3 & 4 & 5 & 6 & 7 \\
\hline (L,F) & 4 & 3 & 2 & 1.5 & 2.5 & 4 & 3.5 \\
(L,S) & 3 & 2.5 & 2 & 3.2 & 2 & 2.5 & 3 \\
(L,V) & 1 & 1.5 & 0.5 & 1 & 0.5 & 2 & 1 \\
(L,C) & 3 & 2.5 & 4 & 3.5 & 3 & 2 & 2.5 \\
(L,M) & 4 & 3 & 3.5 & 3 & 4 & 3 & 3.5 \\
(F,L) & 0.2 & 0.25 & 0.5 & 0.5 & 0.67 & 0.33 & 0.25 \\
(F,S) & 6 & 3.5 & 4 & 4.5 & 5 & 7 & 6.5 \\
(F,V) & 3 & 2.5 & 3 & 4 & 3.5 & 2 & 2.5 \\
(F,C) & 0.2 & 0.25 & 0.33 & 0.2 & 0.17 & 0.2 & 0.25 \\
(F,M) & 1 & 1.5 & 1 & 2 & 1.5 & 0.5 & 2 \\
(S,L) & 0.5 & 0.33 & 0.5 & 0.33 & 0.33 & 0.5 & 0.33 \\
(S,F) & 0.2 & 0.25 & 0.33 & 0.2 & 0.17 & 0.17 & 0.17 \\
(S,V) & 0.2 & 0.25 & 0.2 & 0.25 & 0.33 & 0.2 & 0.25 \\
(S,C) & 0.17 & 0.25 & 0.2 & 0.33 & 0.5 & 0.2 & 0.33 \\
(S,M) & 0.2 & 0.25 & 0.33 & 0.25 & 0.25 & 0.2 & 0.33 \\
(V,L) & 0.5 & 0.5 & 3 & 0.5 & 2 & 0.5 & 0.5 \\
(V,F) & 0.5 & 0.33 & 0.5 & 0.2 & 0.25 & 0.5 & 0.33 \\
(V,S) & 4 & 3 & 4 & 4 & 3 & 4 & 3 \\
(V,C) & 1 & 0.5 & 2 & 0.5 & 1 & 1.5 & 2 \\
(V,M) & 0.33 & 0.5 & 0.5 & 0.33 & 0.5 & 1 & 0.5 \\
(C,L) & 0.5 & 0.33 & 0.25 & 0.33 & 0.5 & 0.33 & 0.5 \\
(C,F) & 4 & 3 & 2 & 4 & 5 & 5 & 3 \\
(C,S) & 5 & 3 & 5 & 2 & 3 & 4 & 3 \\
(C,V) & 1 & 2 & 0.5 & 3 & 0.5 & 0.5 & 0.33 \\
(C,M) & 3 & 2 & 2.5 & 3.5 & 3 & 2 & 3.5 \\
(M,L) & 0.2 & 0.5 & 0.25 & 0.25 & 0.25 & 0.5 & 0.33 \\
(M,F) & 1 & 0.5 & 0.5 & 0.33 & 0.5 & 3 & 0.33 \\
(M,S) & 4 & 3 & 4 & 3 & 5 & 4 & 3 \\
(M,V) & 4 & 3 & 2 & 4 & 3 & 1 & 3 \\
(M,C) & 0.33 & 0.33 & 0.5 & 0.25 & 0.33 & 0.5 & 0.25 \\
\hline
\end{tabular}

Table 2. Comparisons Data of three schools with respect to Learning; $N=5$.

\begin{tabular}{|c|ccccc|}
\hline \multirow{2}{*}{ Pairs } & \multicolumn{5}{|c|}{ Observations } \\
\cline { 2 - 6 } & 1 & 2 & 3 & 4 & 5 \\
\hline (A,B) & 0.33 & 0.5 & 0.33 & 0.5 & 1 \\
(A,C) & 0.5 & 0.33 & 0.33 & 0.25 & 0.33 \\
(B,A) & 2 & 3 & 3 & 2 & 1 \\
(B,C) & 3 & 3.5 & 2 & 2.5 & 3 \\
(C,A) & 3 & 4 & 2 & 3 & 2 \\
(C,B) & 0.5 & 0.25 & 0.33 & 0.5 & 0.25 \\
\hline
\end{tabular}


Table 3. Comparisons Data of three schools with respect to Friends; $\mathbf{N}=6$.

\begin{tabular}{|c|cccccc|}
\hline & \multicolumn{7}{|c|}{ Observations } \\
\cline { 2 - 7 } Pairs & 1 & 2 & 3 & 4 & 5 & 6 \\
\hline (A,B) & 1 & 1.5 & 1 & 1 & 0.5 & 1 \\
(A,C) & 1 & 2 & 1.5 & 0.5 & 1 & 0.5 \\
(B,A) & 1 & 0.5 & 0.5 & 1 & 2 & 1 \\
(B,C) & 1 & 1 & 1 & 1 & 0.5 & 1 \\
(C,A) & 1 & 0.33 & 0.5 & 3 & 1 & 3 \\
(C,B) & 1 & 0.5 & 1 & 0.5 & 3 & 1 \\
\hline
\end{tabular}

Table 4. Comparisons Data of three schools with respect to School Life $\mathbf{N}=6$.

\begin{tabular}{|c|cccccc|}
\hline & \multicolumn{7}{|c|}{ Observations } \\
\cline { 2 - 7 } Pairs & 1 & 2 & 3 & 4 & 5 & 6 \\
\hline (A,B) & 5 & 4.5 & 5 & 5.5 & 4.5 & 5 \\
(A,C) & 1 & 1 & 0.5 & 2 & 1 & 1 \\
(B,A) & 0.25 & 0.2 & 0.25 & 0.2 & 0.2 & 0.25 \\
(B,C) & 0.2 & 0.25 & 0.2 & 0.2 & 0.33 & 0.25 \\
(C,A) & 1 & 0.5 & 2 & 0.33 & 1 & 0.5 \\
(C,B) & 4 & 3 & 5 & 4 & 3 & 5 \\
\hline
\end{tabular}

Table 5. Comparisons Data of three schools with respect to Vocational Training; $N=5$.

\begin{tabular}{|c|c|c|c|c|c|}
\hline \multirow[b]{2}{*}{ Pairs } & \multicolumn{5}{|c|}{ Observations } \\
\hline & 1 & 2 & 3 & 4 & 5 \\
\hline$(\mathrm{A}, \mathrm{B})$ & 9 & 8 & 8.5 & 9.5 & 7.5 \\
\hline$(\mathrm{A}, \mathrm{C})$ & 7 & 6.5 & 7.5 & 7.5 & 7 \\
\hline$(\mathrm{B}, \mathrm{A})$ & 0.1 & 0.11 & 0.12 & 0.11 & 0.10 \\
\hline$(\mathrm{B}, \mathrm{C})$ & 0.2 & 0.25 & 0.2 & 0.33 & 0.25 \\
\hline$(\mathrm{B}, \mathrm{A})$ & 0.1 & 0.11 & 0.12 & 0.11 & 0.10 \\
\hline$(\mathrm{C}, \mathrm{A})$ & 0.17 & 0.14 & 0.12 & 0.17 & 0.14 \\
\hline$(\mathrm{C}, \mathrm{B})$ & 4 & 3 & 4 & 2 & 5 \\
\hline
\end{tabular}

Table 6. Comparisons Data of three schools with respect to College Preparation; $\mathrm{N}=7$.

\begin{tabular}{|c|ccccccc|}
\hline & \multicolumn{7}{|c|}{ Observations } \\
\cline { 2 - 8 } Pairs & 1 & 2 & 3 & 4 & 5 & 6 & 7 \\
\hline (A,B) & 0.5 & 0.33 & 1 & 0.5 & 0.33 & 1 & 1 \\
(A,C) & 1 & 1 & 2 & 1 & 0.5 & 0.5 & 1 \\
(B,A) & 3 & 4 & 1 & 3 & 4 & 1 & 1 \\
(B,C) & 2 & 1.5 & 1 & 1.5 & 2 & 1.5 & 1.5 \\
(C,A) & 1 & 0.5 & 0.33 & 1 & 3 & 3 & 1.5 \\
(C,B) & 0.5 & 0.5 & 0.5 & 0.5 & 0.33 & 0.5 & 1 \\
\hline
\end{tabular}


Table 7. Comparisons Data of three schools with respect to Music Classes $\mathbf{N}=5$.

\begin{tabular}{|c|ccccc|}
\hline & \multicolumn{5}{|c|}{ Observations } \\
\cline { 2 - 6 } Pairs & 1 & 2 & 3 & 4 & 5 \\
\hline (A,B) & 6 & 5.5 & 6.5 & 4.5 & 5 \\
(A,C) & 4 & 4.5 & 3.5 & 5 & 4.5 \\
(B,A) & 0.14 & 0.18 & 0.14 & 0.2 & 0.25 \\
(B,C) & 0.33 & 0.5 & 0.33 & 0.33 & 0.5 \\
(C,A) & 0.33 & 0.2 & 0.25 & 0.2 & 0.25 \\
(C,B) & 3 & 3 & 4 & 4 & 3 \\
\hline
\end{tabular}

The estimates $p^{\prime}, p^{L \prime}, p^{F \prime}, p^{S \prime}, p^{V \prime}, p^{C \prime}, p^{M \prime}$ respectively for $\pi^{\prime}, \pi^{L \prime}, \pi^{F \prime}, \pi^{S \prime}, \pi^{V \prime}, \pi^{C \prime}, \pi^{M \prime}$ are computed using (5) are given by:

$$
\begin{aligned}
& p^{\prime}=(0.33,0.14,0.06,0.14,0.20,0.13) \\
& p^{L \prime}=(0.17,0.57,0.26) \\
& p^{F \prime}=(0.34,0.32,0.34) \\
& p^{S \prime}=(0.48,0.11,0.41) \\
& p^{V \prime}=(0.80,0.07,0.13) \\
& p^{C \prime}=(0.27,0.47,0.26) \\
& p^{M \prime}=(0.70,0.11,0.19)
\end{aligned}
$$

The estimate $p^{*}=\left(p_{A}^{*}, p_{B}^{*}, p_{C}^{*}\right)$ of the over-all priority weight vector $\pi^{*}$ is then obtained through (1) as $(.3895, .3576, .2529)$.

We have constructed the artificial data in Tables 1 through 7 as random fluctuations around the example given in (Saaty, 1980) collected from a single indivudual. These data in Tables 1 through 7 were used by Basak, 1989 in a separate statistical model. We were surprised to see that the estimates in () were very close to the answers obtained in (Basak, 1989). These values are also very close to the solution of Saaty'e eigenvalue method solution. In this article, the methods used were very simple and less time consuming. Yet the solutions are very close to the ones used in other methods.

Next we find the estimates of the covariance matrices of the estimates of $p, p^{L}, p^{F}, p^{S}, p^{V}, p^{C}$ and $p^{M}$ using (6) respectively as follows.

Estimated covariance matrix of $p$ :

$$
S=\left[\begin{array}{cccccc}
0.0038 & -0.0008 & -0.0002 & -0.0013 & 0.0001 & 0.0010 \\
-0.0008 & 0.0003 & 0.0001 & 0.0005 & -0.0003 & -0.0002 \\
-0.0002 & 0.0001 & 0.0000 & 0.0001 & -0.0000 & -0.0001 \\
-0.0013 & 0.0005 & 0.0001 & 0.0021 & -0.0002 & -0.0007 \\
0.0001 & -0.0003 & -0.0000 & -0.0002 & 0.0009 & -0.0002 \\
0.0010 & -0.0002 & -0.0001 & -0.0007 & -0.0002 & 0.0006
\end{array}\right]
$$


Estimated covariance matrix of $p^{L}$ :

$$
S_{L}=\left[\begin{array}{ccc}
0.0005 & -0.0009 & -0.0007 \\
-0.0009 & 0.0046 & 0.0005 \\
-0.0007 & 0.0005 & 0.0034
\end{array}\right]
$$

Estimated covariance matrix of $p^{F}$ :

$$
S_{F}=\left[\begin{array}{ccc}
0.0076 & -0.0026 & -0.0079 \\
-0.0026 & 0.0017 & 0.0026 \\
-0.0079 & 0.0026 & 0.0106
\end{array}\right]
$$

Estimated covariance matrix of $p^{S}$ :

$$
S_{S}=\left[\begin{array}{ccc}
0.0080 & -0.0003 & -0.0100 \\
-0.0003 & 0.0001 & 0.0002 \\
-0.0100 & 0.0002 & 0.0167
\end{array}\right]
$$

Estimated covariance matrix of $p^{V}$ :

$$
S_{V}=\left[\begin{array}{ccc}
0.0001 & 0.0000 & 0.0001 \\
0.0000 & 0.0000 & -0.0000 \\
0.0001 & -0.0000 & 0.0002
\end{array}\right]
$$

Estimated covariance matrix of $p^{C}$ :

$$
S_{C}=\left[\begin{array}{ccc}
0.0065 & -0.0066 & 0.0006 \\
-0.0066 & 0.0095 & -0.0019 \\
0.0006 & -0.0019 & 0.0052
\end{array}\right]
$$

Estimated covariance matrix of $p^{M}$ :

$$
S_{M}=\left[\begin{array}{ccc}
0.0000 & 0.0000 & -0.0000 \\
0.0000 & 0.0005 & -0.0003 \\
-0.0000 & -0.0003 & 0.0008
\end{array}\right] .
$$

Finally, the estimated covariance matrix of the estimated over-all priority vector $p^{*}$ is obtained through (15) as:

$$
\left[\begin{array}{ccc}
0.0012 & -0.0008 & -0.0023 \\
-0.0008 & 0.0045 & -0.0016 \\
-0.0023 & -0.0016 & 0.0053
\end{array}\right]
$$


Next, we apply the statistical inference methods developed in this article to the hierarchy of the six criteria. First we test whether the priority vector $\boldsymbol{\pi}$ of the criteria is given by vector $\boldsymbol{\pi}_{0}=(.3, .14, .1, .13, .2, .13)$; i.e, we test the hypothesis $H_{01}: \boldsymbol{\pi}=\boldsymbol{\pi}_{0}$. The test statistic $T_{1 N}^{2}=N\left(\overline{\mathbf{Y}}_{N}-\boldsymbol{\pi}_{0}\right)^{\prime} \mathbf{S}_{N}^{-1}\left(\overline{\mathbf{Y}}_{N}-\boldsymbol{\pi}_{0}\right)$, given in section 3.1, for testing $H_{01}$ is computed to be 1243.0966. The critical value $T_{10}^{2}=\frac{(N-1)(s)}{N-s} F_{s, N-s}(\alpha)$ given by $(7)$ is computed as $7 \times 6 F_{6,1}(.05)=9828$. Therefore, in this case, $H_{01}$ is not rejected at $5 \%$ level of significance since 1243.0966 is not larger than 9828 and we believe that the priority vector of the six criteria is given by $(.3, .14, .1, .13, .2, .13)$.

Secondly, we test the rank order in the priority vector $\boldsymbol{\pi}$ of the criteria. Using (11), simultaneous confidence intervals of $\pi_{L}-\pi_{C}, \pi_{C}-\pi_{F}, \pi_{F}-\pi_{V}, \pi_{V}-\pi_{M}, \pi_{M}-\pi_{S}$ turn out to be $(.07, .19),(.01, .11),(-.03, .31)$, $(-.02, .3),(-.01, .27)$. Since the last three of these confidence intervals contain zero, we conclude the rank order of the priorities of six criteria as $\pi_{L}>\pi_{C}>\pi_{F}=\pi_{V}=\pi_{M}=\pi_{S}$.

In order to apply the methodology developed in the article for comparing the priority of two groups, we considered another artificial comparisons Data of six criteria with respect to overall satisfaction with school for a second group of individuals with again $N=7$ individuals. We are suppressing the details of the data as well as computations for the sake of brevity. The estimated priority vector of those six criteria for the second group was found to be $(0.22,0.14,0.21,0.14,0.13,0.16)$. The equality of the priority vectors is tested by the hypothesis $H_{02}: \boldsymbol{\pi}^{(1)}=\boldsymbol{\pi}^{(2)}$ where $\boldsymbol{\pi}^{(1)}=(.3, .14, .1, .13, .2, .13)$ and $\boldsymbol{\pi}^{(2)}=(0.2,0.15,0.2,0.15,0.15,0.15)$. The test statistic $T_{2 N}^{2}=\frac{N_{1} N_{2}}{N_{1}+N_{2}}\left(\overline{\mathbf{Y}}_{N_{1}}^{(1)}-\overline{\mathbf{Y}}_{N_{2}}^{(2)}\right)^{\prime} \mathbf{S}^{-1}\left(\overline{\mathbf{Y}}_{N_{1}}^{(1)}-\overline{\mathbf{Y}}_{N_{2}}^{(2)}\right)$ given by (??) was computed to be 174.2463 . The critical value $T_{20}^{2}=\frac{\left(N_{1}+N_{2}-2\right)(s)}{N_{1}+N_{2}-s-1} F_{s, N_{1}+N_{2}-s-1}(\alpha)$ given by $(12)$ is computed as $\frac{12 \times 6}{7} F_{6,7}(.05)=39.81$. Since 174.2463 is larger than the critical value $39.81, H_{02}$ is rejected at $5 \%$ level of significance and we believe that the priority vectors of the six criteria for these two groups are not equal.

Since we find that the priority vectors of two groups are not equal, we investigate into the simultaneous confidence intervals for the differences of component priorities $\pi_{u}^{(1)}-\pi_{u}^{(2)} ; u=L, F, S, V, C, M$. Using (14), simultaneous confidence intervals of $\pi_{L}^{(1)}-\pi_{L}^{(2)}, \pi_{F}^{(1)}-\pi_{F}^{(2)}, \pi_{S}^{(1)}-\pi_{S}^{(2)}, \pi_{V}^{(1)}-\pi_{V}^{(2)}, \pi_{C}^{(1)}-\pi_{C}^{(2)}, \pi_{M}^{(1)}-\pi_{M}^{(2)}$ turn out to be $(.05, .17),(-.07, .07),(-.23,-.07),(-.09, .09),(.03, .11),(-.07, .01)$. Since the confidence intervals for $\pi_{L}^{(1)}-\pi_{L}^{(2)}, \pi_{S}^{(1)}-\pi_{S}^{(2)}$ and $\pi_{C}^{(1)}-\pi_{C}^{(2)}$ do not contain zero, we conclude that the components $L, S$ and $C$ of the priority vectors of two groups are significantly different.

\section{Concluding Remark}

In this article, we use a simple statistical model to make relevent inferences in AHP which allow for dependence among the entries of the pairwise comparison matrix. Using this model, one can also test for the rank order in the priorities for a group of individuals and test whether there is any significant differences in the prioirity weights between two separate groups of individuals.

\section{REFERENCES}

Arbel, A. (1989). Approximate articulation of preference and priority derivation. European Journal of Operational Research, 43, 317-326.

Basak, I. (1989). Estimation of the multi-criteria worths of the alternatibves in a hierarchical structure of comparisons. Communications in Statistics - Theory and Methods, 18, 3719-3738.

Basak, I. (1991). Inference in pairwise comparison experiments based on ratio scales. Journal of Mathematical Psychology, 35, 8091.

Basak, I. (1998). Probabilistic judgments specified partially in the Analytic Hierarchy Process. European Journal of Operational Research, 108, 153164. 
Bryson, N. \& Joseph, A. (2000). Generating consensus priority interval vectors for group decision-making in the AHP. Journal of Multicriteria Decision Analysis, 9, 127137.

Crawford, G. \& Williams, C.(1985). A note on the analysis of subjective judgment matrices. Journal of Mathematical Psychology, 29, 387405.

de Jong, P.(1984). A statistical approach to Saatys scaling method for priorities. Journal of Mathematical Psychology, 28467478.

Genest, C. \& Rivest, L.P. (1994). A statistical look at Saatys method of estimating pairwise preferences expressed on a ratio scale. Journal of Mathematical Psychology, 38, 477496.

Hahn, E.D. (2003). Decision making with uncertain judgments: A stochastic formulation of the Analytic Hierarchy Process, Decision Sciences, 34, 443466.

Haines, L.M. (1998). A statistical approach to the Analytic Hierarchy Process with interval judgments. (I). Distributions on feasible regions. European Journal of Operational Research, 110112125.

Jensen, R.E. (1984). An alternative scaling method for priorities in hierarchical structures. Journal of Mathematical Psychology, 28, 317332.

Ramanathan, R. (1997). Stochastic decision making using multiplicative AHP. European Journal of Operational Research, 97, 543549.

Saaty, T.L. (1980). The Analytic Hierarchy Process. McGraw-Hill.

Saaty, T.L. \& Vargas, L.G. (1987). Uncertainty and rank order in the Analytic Hierarchy Process. European Journal of Operational Research, 32, 107117.

Stam, A. \& Duarte Silva, A.P. (2003). On multiplicative priority rating methods for the AHP. European Journal of Operational Research, 145, 92108.

Sugihara,K., Ishii, H. \& Tanaka, H. (2004). Interval priorities in AHP by interval regression analysis. European Journal of Operational Research, 158, 745754. 